\title{
People and a virtual doctor's visit: learning about multiple acceptance aspects of a telemedical scenario
}

\author{
Sarah Mennicken, Oliver Sack, Martina Ziefle \\ Human Technology Centre \\ RWTH Aachen University \\ Aachen, Germany \\ \{mennicken, sack, ziefle\}@humtec.rwth-aachen.de
}

\begin{abstract}
In this paper we report on a study exploring multiple aspects of a virtual doctor's visit as a commonly used example for telemedical services. We compare the conventional doctor's visit with the virtual visit to identify user requirements and needs for the acceptance of such a scenario. In our survey we retrieved data from a total of 93 users between 16 and 67 years. The results of this study describe differences (and similarities) of criteria for choosing a doctor, basic requirements to accept a virtual doctor's visit, and people's opinions on common pro and contra arguments on this scenario. We analyzed the data for potential influences from various user characteristics, such as age, gender, social behavior, or technical competence. Interestingly, most of the reported concerns are not influenced by gender or age, while participants' technical self-confidence is related with various acceptance aspects.
\end{abstract}

Keywords-Medical Technologies, Technology Acceptance, User Study, Doctor's visit, Patient doctor interaction

\section{INTRODUCTION}

Due to the demographic change the number of elderly people that need health care services [1][2] will dramatically increase in the upcoming years. This trend leads to a situation where a decreasing number of doctors will have to care for even more patients with a longer life expectancy and a raised probability for chronic diseases. In addition to an increased need for medical care, there are serious shortcomings as a result of the decreasing number of caregivers and upcoming supply shortfalls regarding societal health insurance funds. Improvements in information and communication technologies (ICT) as well as developments in medical engineering may offer novel or improved opportunities for medical diagnosis, therapy, treatments, and rehabilitation possibilities to master the exigent requirements of an aging society.

Already more than two decades ago computer systems have been installed in most doctor's offices to help accelerating organizational work, and thus caring for more patients. However, patients still have to come into the doctor's office and wait with other people in a waiting room. While sometimes this is inevitable because a physical examination is required, in other cases people wait a long time just to talk a couple of minutes with the doctor even though a personal contact is not necessary from a medical point of view.

\section{RELATED WORK}

Electronic health technologies cover the interaction between patients and health-service providers, institution-to-institution transmission of data, or peer-to-peer communication between patients or health professionals. eHealth technologies also include health information networks, electronic health records, telemedicine services, and personal wearable and portable communication small screen for continuously monitoring patients' health conditions and providing health-related information. Telemedicine possesses the ability to bridge gaps and overcome barriers in a way that was not possible with traditional forms of healthcare [3][4][5][6].

Already in the late 90ies of the last century, early studies considered impact and consequences of telemedical services for different stakeholders (patients, providers, policy and society) [7][8][9]. Since that time, the power of mobile communication technologies improved dramatically. Mobile technology is entering private spaces and increasingly incorporated in smart homes. Recently, there are research trends for more innovative technology supporting doctors [5]. One example is the virtual doctor's visit, a telemedical scenario, which enables remote virtual consultation hours between doctor and patient. This could possibly meet some of the mentioned shortcomings: patients do not need to visit doctors in person in order to get medical advice, but instead they can choose to communicate with the doctor virtually and clarify routine problems or questions, prior to a possible real consultation. Within literature there is a vivid discussion and a growing interest in potential benefits of teleconsultations in many medical fields (e.g. ophthalmology [11], rehabilitation in stroke care [12], or orthopedics [13]). However, within the public perception, a broad reluctance is prevailing [14]: people seem to have skeptical opinions on telemedicine regardless its potential benefits. Some form their opinions based on what they read in magazines, hear in the news, or see in TV shows and movies. There are a lot of doubts regarding the limits of technology, as for example data safety or fears such as doctors being afraid that a therapy mediated by technology might decrease its quality or even finally lead to their unemployment. The authors of [15] show that the acceptance of telemedicine in doctors is a complex phenomenon, for 
which different aspects have to be considered, as for example the perceived usefulness, social norms, attitudes, perceived behavioral control, and intentions. Nevertheless, there is still an enormous knowledge gap about the public discourse and potential ambivalent attitudes to technology-supported care concepts. Also, insufficient knowledge is present to which extent individual beliefs. (Social) trust in healthcare and technology as well as perceptions of potential benefits and risks are influencing telemedicine's acceptance [16].

\section{A VIRTUAL DOCTOR'S VISIT}

The system for a virtual doctor's visit we briefly mentioned in the introduction was introduced to our participants in more detail by describing a specific situation giving an example of a possible usage:

An elderly patient had a heart attack but is stable now and can be discharged from hospital. However, the doctor needs to adapt the medication with the patient once a week. Instead of going to the doctor's practice the patient can also try a virtual doctor's visit on a wall-sized screen in his home (see Fig. 1). Similar to a videoconference they can talk and interact. The prescribed medication is shipped with a pharmacy service the same afternoon.

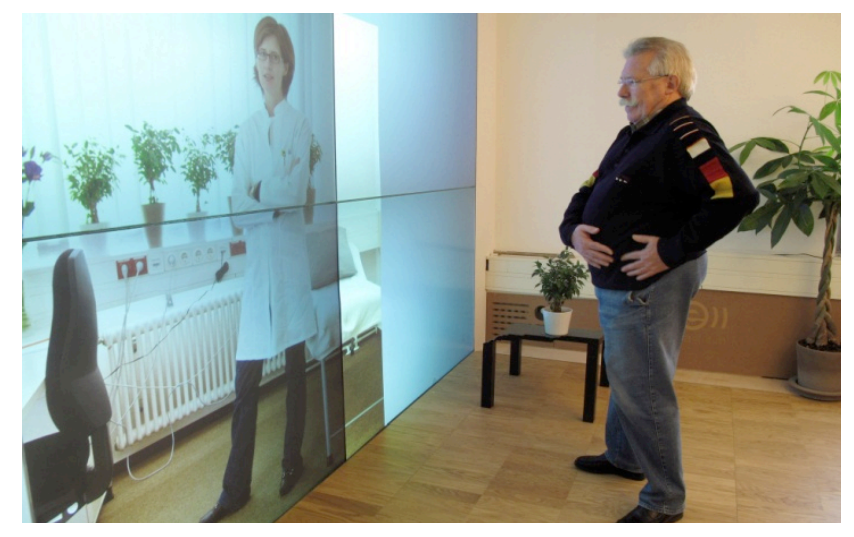

Figure 1. Patient and doctor interacting in a virtual doctor's visit on a wallsized screen in the patient's living room.

\section{QUESTIONS ADDRESSED AND LOGIC OF EMPIRICAL PROCEDURE}

To approach our questions we had two phases of research studies: an initial phase of qualitative interviews to understand potential users better and a questionnaire based on our findings and further literature review to verify our hypotheses and to further explore other questions.

\section{METHODS}

\section{A. Participants}

A total of 93 adults answered the questionnaire. 31 younger adults (4 males, 27 females) with a mean age of 21.5 years $(S D=2.2$; range: 16-25), 31 middle-aged adults (14 males, 17 females) with a mean age of $31.6(S D=6.4$; range: $26-45)$, and 31 older adults (13 males, 18 females) with a mean age of 56.8 $(S D=5,7$; range: $46-67)$ participated. The younger participants were mostly university students of different academic fields (social science, engineering, and informatics). The older and middle-aged participants were highly qualified and covered a broad range of different professions (e.g. administrative officers, secretaries, teachers, clerks, engineers, informatics, and consultants). Most of the older adults participating were active parts of the work force, mentally fit and not hampered by strong age-related sensory and psychomotor limitations. Participants did not contact their physician frequently $(\mathrm{N}=531$ 2 times per year). Half of participants were singles, the other half lived in a partnership. All had at least some computer experience, but were medical technologies novices. Participants did not use a broad range of medical technologies (e.g. hearing aid or blood sugar monitoring device). Only the blood pressure monitoring device was used by the majority of participants, but not more than 1-2 times per month $(\mathrm{N}=24)$. Additionally, we gathered social contact intensity, technical self-confidence, dealing with health, emphasize on usage or ownership of new technologies, importance of technical innovations. With a $\mathrm{M}=$ 4.8 of $10(S D=1.8)$ participants did have neither intensive nor weak social contact to other people. Their technical selfconfidence (STC) was high with a mean score of 71 of 100 $(S D=16)$. Participants had a high awareness in the context of dealing with health $(\mathrm{M}=17.8$ of $24 ; S D=3)$. The emphasis on usage or ownership of new technologies in general was indifferent $(\mathrm{M}=28.1$ of $48 ; S D=8.2)$. The emphasis of on usage or ownership of new technologies in view of medical technologies was judged lower-than- average $(M=2.5$ of 6 ; $S D=1.7)$. In contrast importance of technical innovations in general was increased $(\mathrm{M}=33$ of $48 ; S D=6.5)$. The importance of technical innovations in context of medical technologies strikingly increased $(\mathrm{M}=5.3$ of $6 ; S D=1.1)$.

\section{B. Variables}

1) Independent variables: The first variable is users' age. Contrasting younger participants $(\mathrm{N}=31,16-25 \mathrm{yrs})$, middleaged adults $(\mathrm{N}=31,26-45$ yrs $)$ and older adults $(\mathrm{N}=31,46-67$ yrs). The second independent variable refers to the users' gender. The third variable contains the degree of social contact intensity created by three items summarized to a total score with a maximum chararcteristic of 13 points. This maximum means the participant expressed having frequent social contacts by our statements (e.g. "How often do you meet other people for conversation."). The fourth variable assessed how participants are dealing with health based on four items summarized to a total score with maximum chararcteristic of 24 points when fully agreeing to the presented items (e.g. "I always act on the physician's advice."). The maximum score expresses having a very high attention on their health and a very active participitation in their health care. The fifth variable was the criterion appreciation of technical innovations (ATI) based on eight items summarized to a total score with maximum chararcteristic of 48 points meaning having a high apprecitiation of technical innovation. Additionally, we examined participants' technical self-confidence (STC) based on eight items with a summarized maximum chararcteristic of 100 points meaning having a strong technical self-confidence, i.e. the degree of confidence to which a person believes in own ability to solve technical problems. 
2) Dependent variables: As dependent variables three item clusters of criteria were surveyed: criteria for choosing a doctor (regular doctor's visit versus virtual doctor's visit), requirements for a virtual visit (e.g. "A virtual doctor's visit must be easy to use.") and pro/contra arguments for a virtual visit (e.g. "It increases the quality of the treatment.").

\section{Preliminary study}

Before constructing the questionnaire instrument, we conducted semi-structured interviews with eight elderly participants (with different health states, i.e. either mobile healthy, or living in day or full care in retirement homes). In addition, we interviewed two doctors to contrast the answers of participants with a professional perspective. Participants answered questions concerning organization of a doctor's visit (e.g. "Do you need support by a relative to arrive at the doctor's office?"), doctor's treatment in her/his office (e.g. "Which devices are used to get a diagnosis?"), behavior of the doctor (e.g. "Does the doctor provide an opportunity for private conversation?"), which expectations patients have and which factors influence their acceptance of a visit. Acceptance factors were assessed with the help of the critical incident technique [16] (e.g. "Can you remember a well satisfying doctor's visit? Please describe briefly the situation and satisfying aspects. What kind of behavior showed the doctor?"). According to which target group (e.g. mobile healthy vs. in full care or patient vs. doctor) the interview questions were adapted to the context.

\section{Development of the questionnaire and data collection}

The questionnaire was elaborated from extensive literature research [5][17][18][19][20] and findings of the previously realized semi-structured interviews. The questionnaire was divided into two parts: the first part comprised questions about demographical data, health status, dealing with disease, usage of medical technologies, social contact intensity, importance of technology and technological innovation, and technical selfconfidence. The second part dealt with factors of acceptance in a regular doctor's visit scenario and in a virtual doctor's scenario. In the first instance participants judged general attitudes to their doctor and criteria for choosing a doctor in a regular doctor's visit. After that participants were introduced into the virtual doctor's visit by a scenario of telemedical cardiac disease treatment in patients home environment (see Fig. 1). After a brief description of the scenario the last part covered criteria for choosing a doctor as well as requirements for a virtual visit and pro/contra arguments for a virtual visit. All participants filled in an online questionnaire or paperpencil version with bounded response scales. Scales formats were for choosing a doctor on a scale from 1 ("unimportant") to 7 ("very important") and requirements and pro and contra arguments on a scale from 1 ("fully disagree") to 6 ("fully agree"). Various responses could be annotated with openended comments.

\section{E. Statistical Analysis}

Considering ratings should be analyzed nonparametrically, we were interested in interacting effects, which only can be assessed by analysis of variance procedures.
Therefore results were analyzed by univariate analyses of variance assessing effects of age (divided in the three groups of younger, middle-aged, and older adults) and by using the ttest for gender effects. To be on the safe side not "overestimating" the significance of outcomes, we checked that main effects yield the same significance in both, parametric and non-parametric testing procedures. Additionally Spearman correlations were run with for age, gender, social contact intensity, dealing with health, appreciation of technical innovation, and technical selfconfidence. To compare items for choosing a doctor (regular vs. virtual) paired-sample t-tests and Spearman correlations were run. The significance level was set at $\mathrm{p}<.05$.

A qualitative strategy was adopted to analyze the openended comments. In a first step, all comments were read to extract general impressions and classification categories. Criteria for choosing a doctor in regular and virtual session were grouped in 14 broad categories: competence, doctor's personality, proximity, office rooms, fast service, time, trust, behavior, reputation, result of therapy, recommendation, diligence, kind of specialization, being informed. In a second step, multiple response analysis assessed frequencies of responses.

\section{RESULTS}

In the following chapter we are going to present multiple acceptance facets of a simple scenario in an intelligent home environment to identify and understand acceptance problems from a users perspective. First, we describe how people choose their doctors for a regular doctor's visit and compare it to what criteria they would have to choose a doctor for a virtual visit. Then we describe general requirements people stated to have for accepting a virtual visit. The last part of the result analyzes how people rated common pro/contra arguments and their opinions expressed in open-ended comments.

\section{A. Choosing a doctor}

The data described is retrieved from three ranked opencomment fields on participants' most important criteria to pick a doctor. We coded the comments into categories and evaluated them quantitatively. Overall we received 237 mentions by 93 people and we report on the five most mentioned codes (see Fig. 2).

$26.2 \%$ of comments addressed "competence", $74.7 \%$ of our participants mentioned it in general $\left(1^{\text {st }}, 2^{\text {nd }}\right.$, or $3^{\text {rd }}$ criteria $)$, $44.4 \%$ as the most important criteria. "Doctor's personality" as a selection criterion was mentioned by $16.5 \%$ of all mentions, and by $47 \%$ of all people. $14.3 \%$ of the mentions were about having the practice close or convenient to their home or work, stated by $41.0 \%$ of the participating people. The doctor having good availability, i.e. getting an appointment quickly, was $9.3 \%$ of all mentions, stated by $26.5 \%$ of people. Trust in the doctor was $8.0 \%$ of all mentions with $22.9 \%$ of people reporting it. 


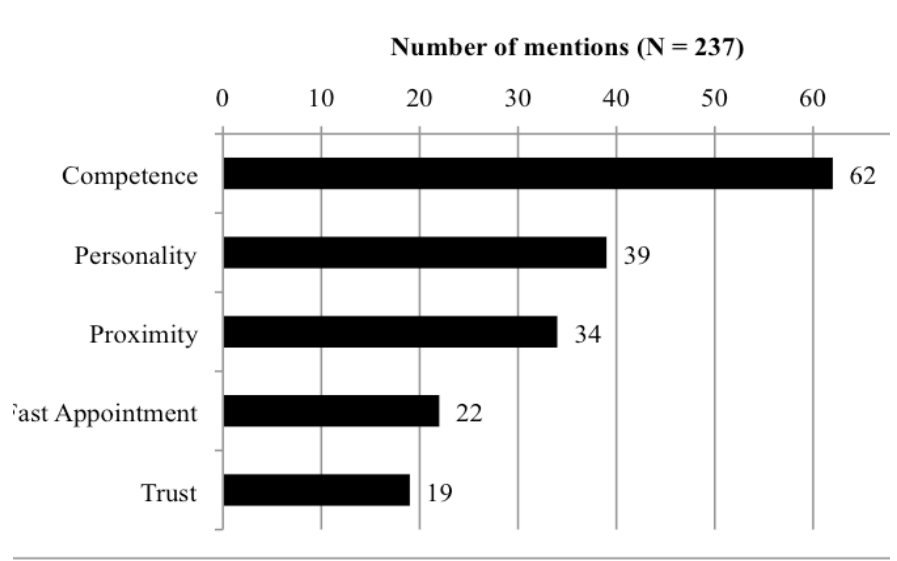

Figure 2. Five most mentioned criteria for picking a doctor.

Further we compared how people choose a doctor for a regular doctor's visit with how they would choose a doctor for a virtual visit. We found significant differences in the two criteria: "proximity" and "recommendation". The importance of having the doctor close to home sunk for the virtual visit $(\mathrm{t}(80)=10.2, \mathrm{p}=0.0 ; \mathrm{M}($ classic $)=5.6, \mathrm{M}($ digital $)=3.1$, see Fig. 3$)$. Also, the importance of having a recommendation for doctor in a virtual doctor's visit was reported as less important than for a regular visit (see Fig. 3).

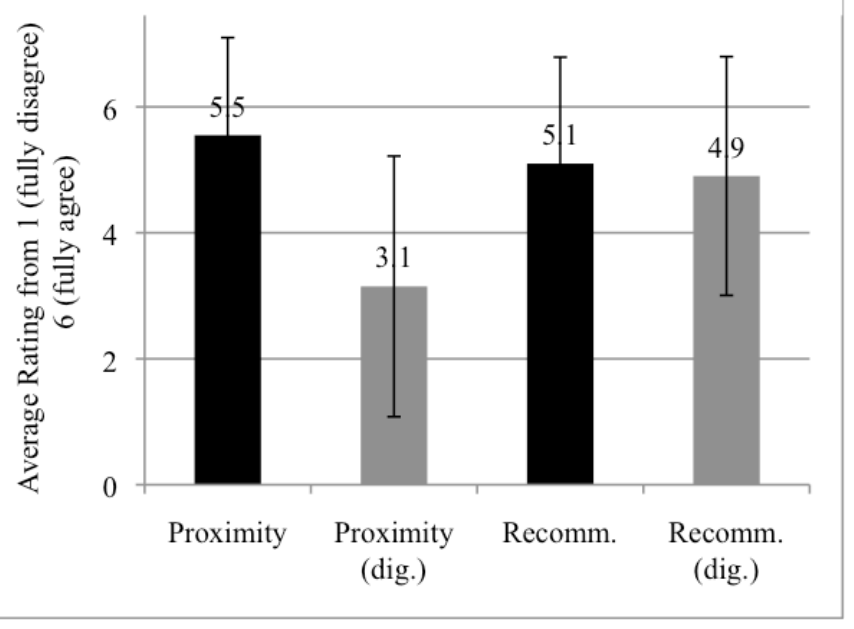

Figure 3. Significant differences between choosing a doctor for a regular (black-colored) or virtual doctor's visit (grey-colored)

For the other criteria, the virtual visit was not differently evaluated than a conventional consultation (see Fig. 4). Thus, the different scenarios did not influence the importance of the doctor being an expert in his field, taking enough time for each patient, not having to wait for long, and getting an appointment quickly.

\section{1) Effect of age and gender}

In order to learn if the ratings were impacted by the age or gender of participants, ANOVA analyses were run. In contrast to outcomes in many other fields of technology usage (e.g., [21][22]), in which there are remarkable differences between younger and older adults, this is not the case in this context. Age did not play a major role for the evaluations of telemedical consultations. The only finding we made regarding the age affect was a weak positive correlation between age and the importance of the doctor taking time for the patient in the regular doctor's visit $(\mathrm{r}=.2, \mathrm{p}<.05)$.

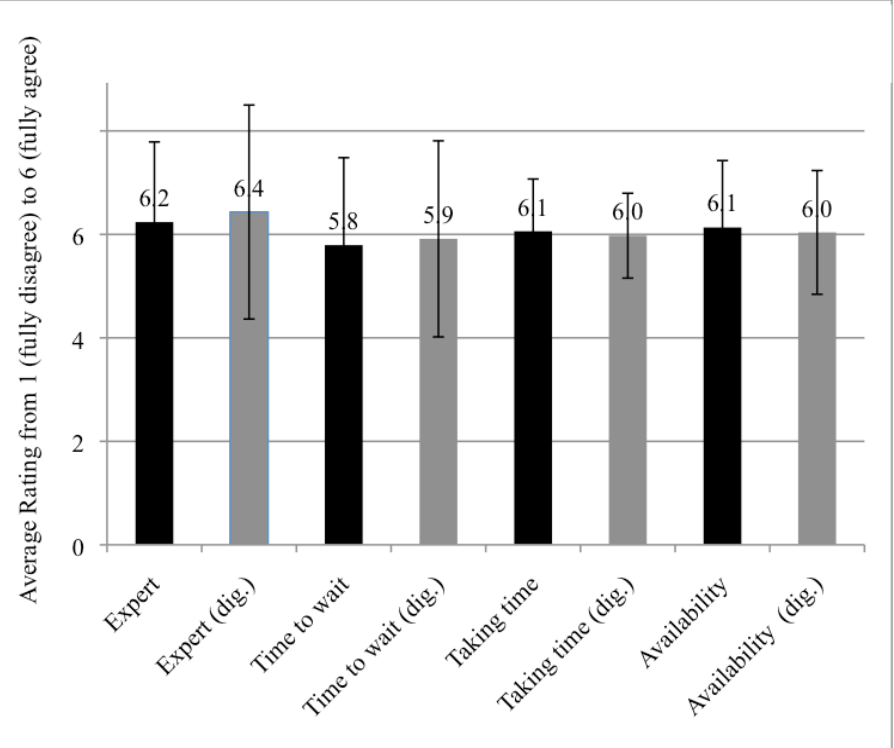

Figure 4. Similar opinions on criteria for choosing a doctor for a regular (black-colored) or virtual visit (grey colored)

Also, there also was no noticeable gender effect. Women and men only differed in one question about requirements for their doctor: for women $(\mathrm{M}=5.6 ; \mathrm{SD}=0.5)$ it is more important than for men $(\mathrm{M}=5.1 ; \mathrm{SD}=1.3)$ that they do not have to be ashamed of anything in front of them $(\mathrm{t}(34.72)=-2 ; \mathrm{p}<.05)$.

\section{B. Requirements for a virtual visit}

We further asked participants' about their requirements to use a virtual visit with their doctor by having them rating eleven statements on a scale from 1 ("fully disagree") to 6 ("fully agree"). The rating was done in order to see general tendencies and some statements were redundant to check the integrity of results. After rating them they were asked to choose three statements that they consider most important to find out perceived key issues and concerns

Overall participants judged "data safety" (67,9\%; "My medical data is kept safe from unauthorized access.") as the most important requirement to use the virtual visit. "Covering of the costs" (58\%, "My insurance covers the costs for a virtual doctor's visit completely") is the second most important criteria followed by "data access" (53\%; "Only my doctor has access to my medical data"). Least important are recommendation (3,7\%; "Using virtual visit on the recommendation of my doctor.") and fun (3,7\%; "Using the system must be pleasant.") (see Fig. 5) 
TABLE I. ITEMS: REQUIREMENTS FOR USING VIRTUAL VISIT TO BE APPROVED OR DISAPPROVED ON A SIX POINT SCALE ( $1=$ FULLY DISAGREE TO 6=FULLY AGREE)

In order to use the virtual visit with my doctor, following requirements must be met...

Technology is on the back seat during treatment.

Only my doctor has access to my medical data.

My medical data is kept safe from unauthorized access.

I can speak with my doctor about personal topics.

Using virtual visit on the recommendation of my doctor.

My insurance covers the costs for a virtual doctor's visit completely.

My Doctor gives me free choice of treatment method.

A doctor's appointment for virtual visit is easier to organize than a conventional visit.

Learning to operate the system is easy for me.

Overall, virtual visit is easy to use.

Using the system must be pleasant.

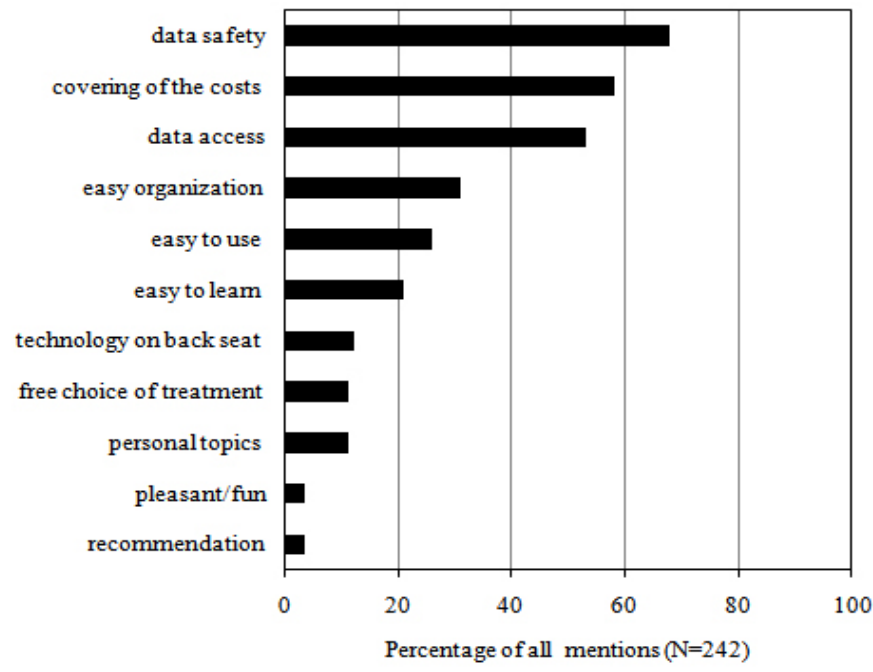

Figure 5. Mentions of requirements for virtual visit.

\section{1) Effect of age and gender}

Fig. 6 illustrates the statistic connections between the relevant requirements items and the user factors. As can be seen for age and gender there are only two statistically significant relations. First age and talking about personal topics with the doctor revealed a significant correlation $(r=.22 ; \mathrm{p}<0.05)$. With higher age it is more important to talk about personal things. Second gender and having a free choice of treatment method showed significant correlation $(\mathrm{r}=.26 ; \mathrm{p}<0.05)$. For women it is significantly more important to have free choice about treatment method.

The ANOVA showed that different age groups did not significantly differ in their judgment of requirements. However, gender effects were found: women reached a higher score in data safety $(M=5.8 ; S D=0.5)$ than males $(M=5.7$; $\mathrm{SD}=0.7)(\mathrm{t}(79)=-1,2 ; \mathrm{p}<0.05)$. As mentioned earlier in the report of correlations for women it is significantly more important to have free choice about treatment method $(\mathrm{M}=4.1$; $\mathrm{SD}=1.5)$, than for male participants $(\mathrm{M}=3.2 ; \mathrm{SD}=1.5)(\mathrm{t}(79)=-$ $2.45 ; \mathrm{p}<0.05)$. Furthermore female respondents ascribe significantly more importance to an easier organization of a virtual visit $(\mathrm{M}=4.9 ; \mathrm{SD}=1.4)$ than male $(\mathrm{M}=5.0 ; \mathrm{SD}=0.8)$ $(\mathrm{t}(80)=.39 ; \mathrm{p}<0.05)$. Additionally, a marginally significant effect $(\mathrm{p}<.1)$ was found for the wish to speak with the doctor about personal topics, which was rated as more important for women $(\mathrm{M}=4.5 ; \mathrm{SD}=1.4)$ than for men $(\mathrm{M}=5.0 ; \mathrm{SD}=0.8)$ $(\mathrm{t}(79)=-1.34 ; \mathrm{p}<0.1)$. To sum up, contrary to men, data safety issues and the personal communication with the doctor about personal topics are more important for women. Male participants judged easier organization of a virtual visit higher.

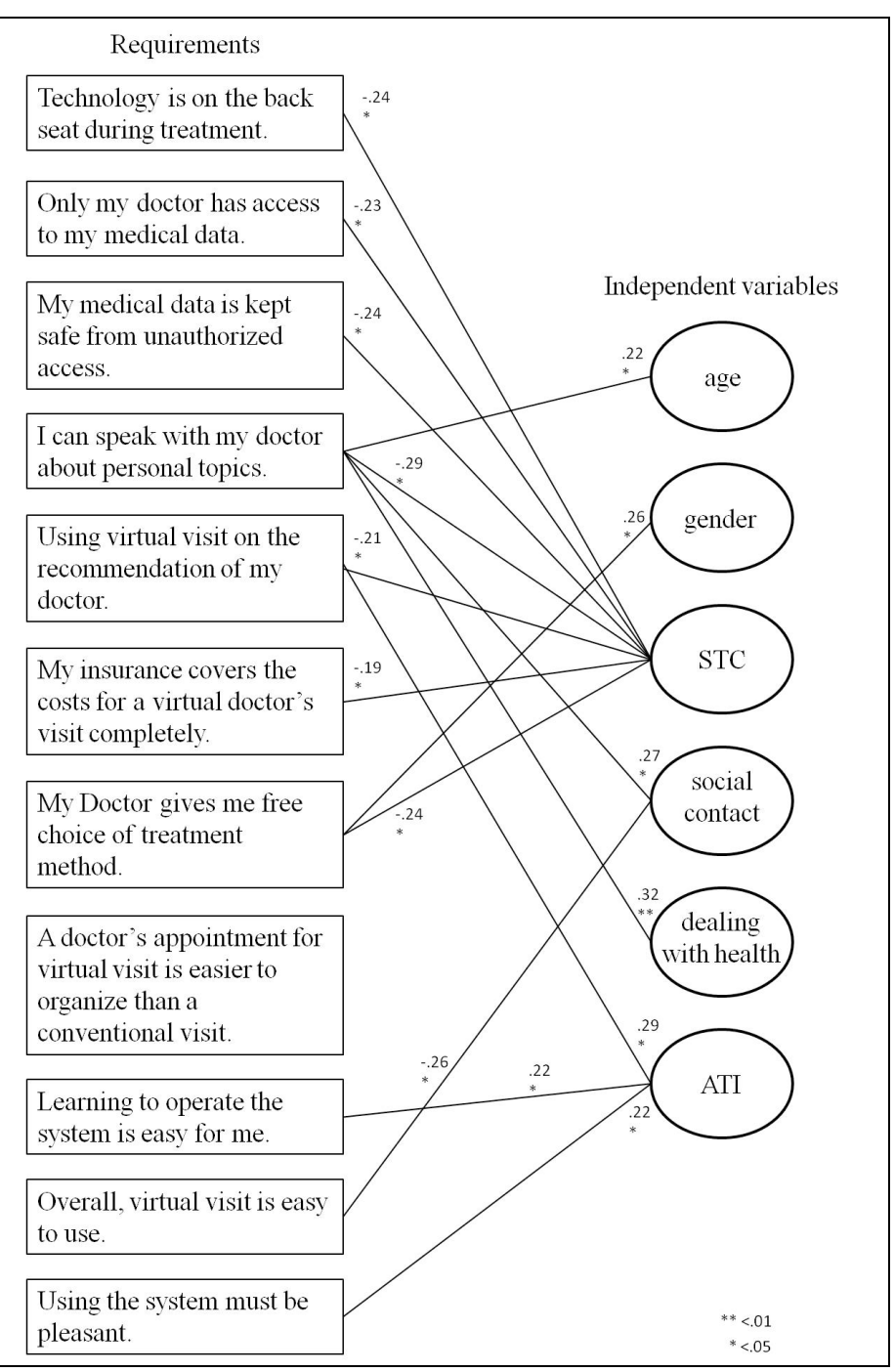

Figure 6. Intercorrelations between requirements for using virtual visit (left) and user factors (right). STC (technical self-confidence). ATI (appreciation of technical innovations).

\section{2) Effects of other individual characteristics}

Beyond age and gender effects, we surveyed other personal factors, which possibly influence the acceptance of telemedicine: technical self-confidence (STC), social contact intensity, dealing with health, and appreciation of technical 
innovation (ATI). According to correlation outcomes, all investigated user factors showed intercorrelations. When looking at the technical self-confidence (STC) nearly all requirements had significant correlations with STC except of the requirements oriented by effort expectancy and performance expectancy known from UTAUT [23]. With higher technical self-confidence most of the requirements (technology on the backseat, data access, data safety, personal topics, recommendation, covering of the costs and free choice) were judged as less important for using the virtual visit. People with a high intensity of social contacts emphasize talking about personal topics and an easy usage of the virtual visit. Additionally, people with a higher score in dealing with health prefer in a markedly way to speak about personal topics than people with a lower score. Participants with a higher ATI stress more a recommendation, easy learning to operate with the system and a pleasant use.

\section{Pro/Contra arguments for a virtual visit}

Within such a complex phenomenon as acceptance for new telemonitoring care concepts, it is insightful to learn the arguments that militate in favor of these new concepts and to understand the nature of the perceived barriers or concerns. To do so, participants had to state the extent of approval or disapproval regarding the pro and the con arguments. For some statements we asked them to share their personal reasons for a specific rating in an extra open-comment field. Then we analyzed the results for age or gender effects. Also correlations with personal variables (e.g. appreciation technical innovation, social contact intensity) are reported.

TABLE II. PRO ITEMS FOR USING VIRTUAL VISIT TO BE APPROVED OR DISAPPROVED ON A SIX POINT SCALE ( 1 =FULLY DISAGREE TO 6 =FULLY AGREE)

It accelerates the doctor's visit and safes time.

It increases the quality of the treatment.

I have to put less effort into organization.

This kind of doctor's visit can be integrated into my daily routines more easily.

I miss the actual physical touch with my doctor e.g. the handshake.

TABLE III. CON ITEMS FOR USING VIRTUAL VISIT TO BE APPROVED OR DISAPPROVED ON A SIX POINT SCALE ( 1 =FULLY DISAGREE TO $6=$ FULLY AGREE)

I refuse that my therapy is administered via technology.

I'm afraid that there might be problems with the technology.

I doubt that my therapy can be administered via technology.

I do not like that the doctor can see and hear me in my home.

Additionally, participants had to rate the neutral item "I do not have to leave the house." on a six point scale from 1 ("fully disagree") to 6 ("fully agree").

As can be seen in Fig. 7 and Fig. 8, on average, the agreement on the pro arguments is higher than on the contra arguments or the neutral statement.

\section{1) Effect of age and gender}

We only found one statement with an age effect (see Fig. 9). There is significant negative correlation between participants' age and "I do not like that the doctor can see and hear me in my home." $(r=-.29 ; p<0.01)$. Older people rated this statement lower, so it seems that they are less concerned about this possible privacy concern. For two statements we could find gender effects: the rating of "I'm afraid that there might be problems with technology." is significantly related to gender $(\mathrm{r}=.23, \mathrm{p}<0.05)$ and the gender of the participant is significantly related to the rating of "I do not have to leave the house." $(\mathrm{r}=.3, \mathrm{p}<0.01)$.

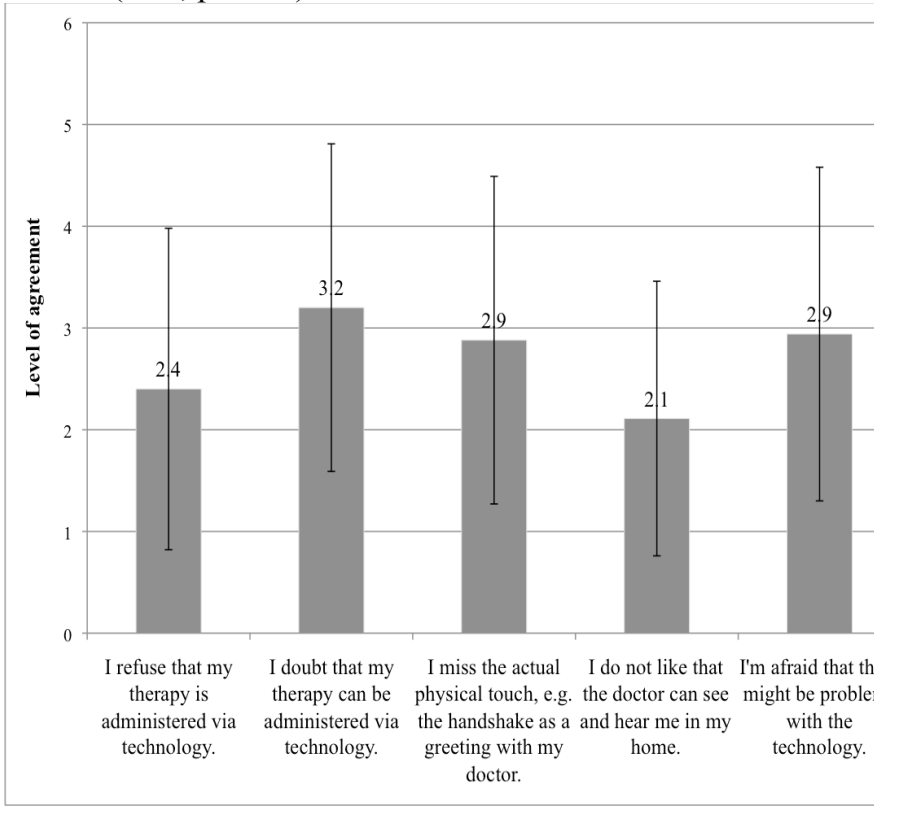

Figure 7. Mean and standard deviation of ratings on contra arguments

2) Effect of other individual characteristics (see Fig. 9) Fig. 9 illustrates that most correlations have been found on negative arguments.

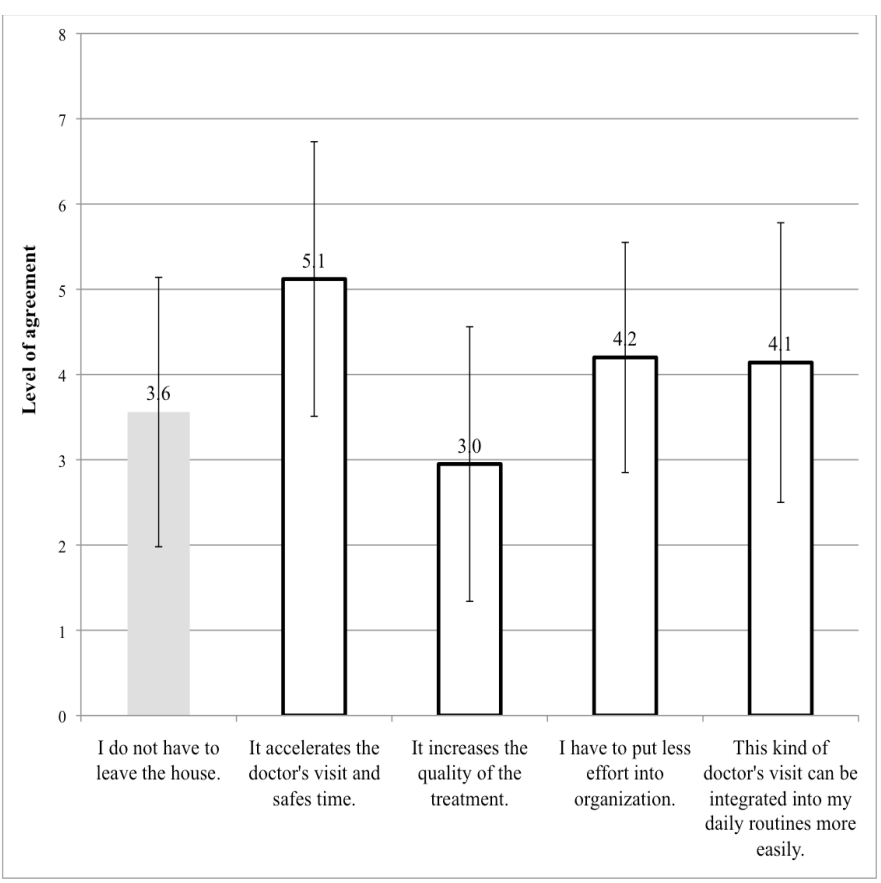

Figure 8. Mean and standard deviation of ratings on pro arguments and the neutral statement 
Not surprisingly, we found that the rating of "I miss the actual physical touch with my doctor, e.g. the handshake" is significantly related to the social contact intensity $(r=.25$; $\mathrm{p}<0.5$ ). This statement is significantly related to the STC ( $r=$.$42 ; \mathrm{p}<0.1)$ as we; $=11$. There is a significant negative correlation between STC and "I'm afraid that there might be problems with the technology." ( $\mathrm{r}=-.61 ; \mathrm{p}<0.01)$, STC and "I doubt that my therapy can be administered via technology." ( $\mathrm{r}=-.28 ; \mathrm{p}<0.5)$, as well as STC and "I refuse that my therapy is administered via technology." ( $\mathrm{r}=-.35 ; \mathrm{p}<0.01)$. The rating of "I do not like that the doctor can see me and hear me in my home." is significantly related to the dealing with health score $(\mathrm{r}=-.25 ; \mathrm{p}<0.5)$, as well as to participants' ATI $(\mathrm{r}=-.29 ; \mathrm{p}<0.5)$. The ATI score is the only characteristic that correlates to pro arguments. It is significantly positively correlated to "I have to put less effort into organization" $(\mathrm{r}=.24 ; \mathrm{p}<0.5)$ and to "This kind of doctor's visit can be integrated into my daily routines more easily."

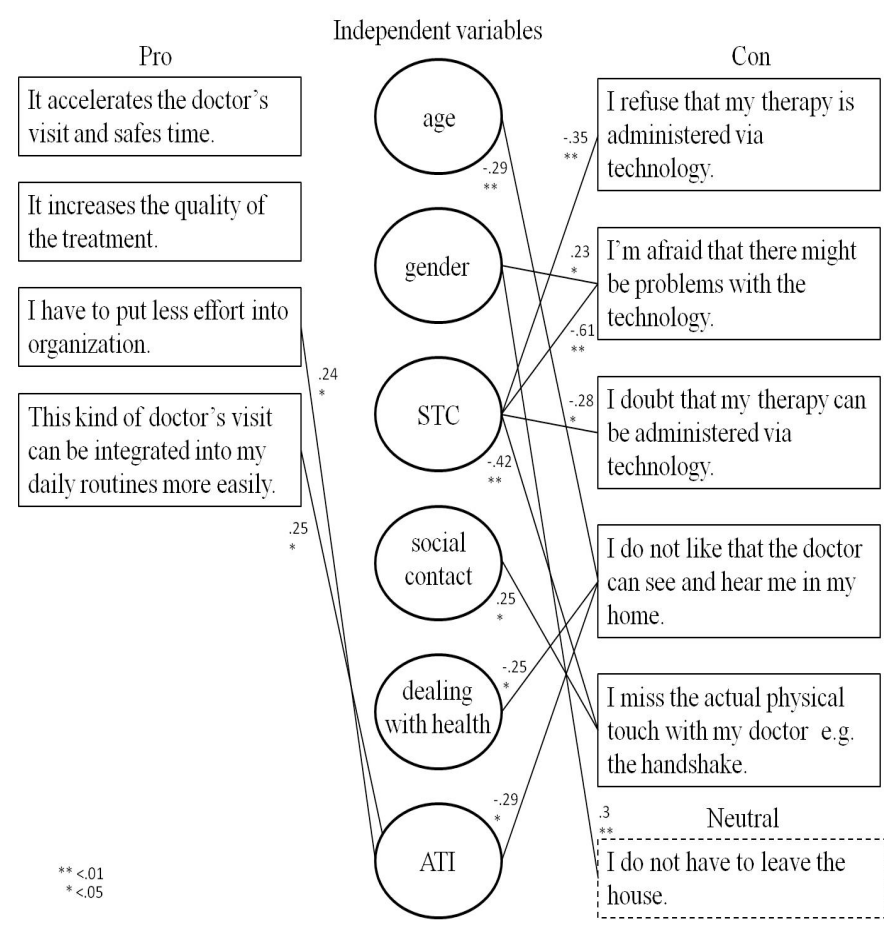

Figure 9. Intercorrelations between pros (left), cons (right), neutral item (lower right), and user factors. STC (technical self-confidence). ATI (appreciation of technical innovations).

We did not find any correlation between participants' characteristics and the following two statements: "It accelerates the doctor's visit and safes time." and "It increases the quality of the treatment." STC seems to be a characteristic that influences people's rating of pro/con arguments mostly, while gender and age do not play a big role.

3) Neutral statement "I do not have to leave the house"

The rating of this item, in contrast to the other items, had two poles in the distribution of the rating (see Fig. 10). To find reasons for this distribution we checked the open-ended comments. People see positive effects of this statement, exemplarily represented by the following participant quote: "Very handy, especially when it is slippery outside or it rains, don't have to use the stairs when I'm impaired". At the same time they also were concerned about social isolation and lack of physical exercise: "Especially for elderly it's important to leave their homes. All communication with people on the way to the doctor (bus/taxi/waiting room/etc.) is lacking. There is no more social interaction. And if elderly people have plenty of one thing - it's time."

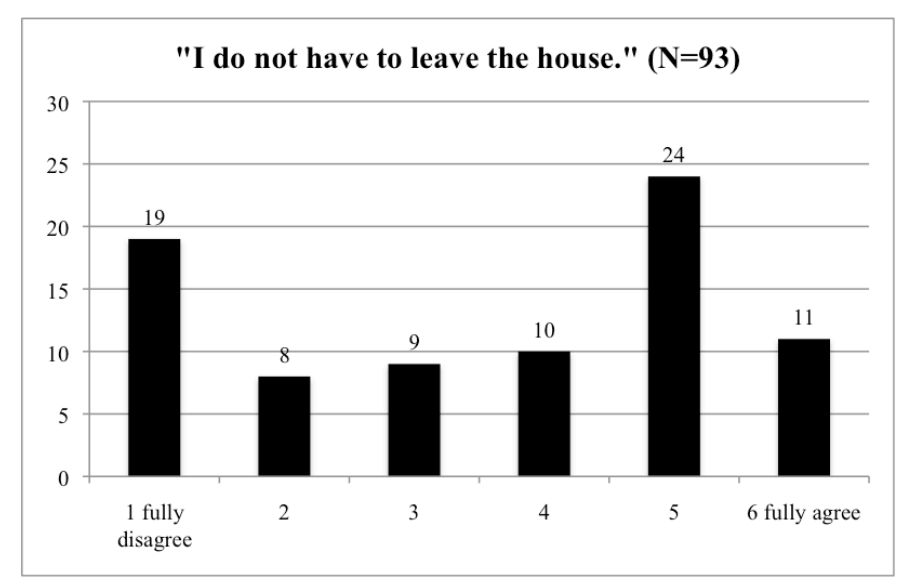

Figure 10. Rating distribution for "I do not have to leave the house"

\section{FUTURE WORK}

The work presented is an approach to broadly explore the complex field of a telemedical scenario. The retrieved data provides us with a background to first, create a suitable system to simulate the scenario in our lab, and second involving more stakeholders besides doctor and patient such as relatives, caregivers, or even insurance companies in our studies. Also, we want to take a further look at the open-ended comments of the participants in our study in order to strengthen our understanding for the individual needs of potential users. Future studies will also have to consider cultural effects, as the acceptance of telemedical applications and virtual doctors' visits might also be influence by different religions and cultural as well as societal values.

\section{CONCLUSION}

The aim of the present study was to explore the acceptance and the perceived barriers of telemedical application, taking a virtual doctor's visit as example. In order to understand the critical requirements of future electronic care concepts and to reflect the kind and nature of hopes and concerns, which determine cognitive mindsets and acceptance patterns towards telemedical applications, a two-step procedure was chosen. First we interviewed selected persons in semi-structured interviews in order to identify the crucial concerns and expectations. Then, 93 users of a wide age range were asked about their criteria for choosing a doctor, contrasting a real and a virtual doctor's visit. Also, we assessed pro and contra arguments for a future usage of such systems. The findings show that-overall-all respondents, independently of age and gender, acknowledge the benefits of telemedical applications. The requirements for a digital doctor are very similar to those for a real one: he/she must be an expert, he/she should take enough time for the consultation and it is important that the time to get an appointment is minimal. However, there are also 
differences between the virtual and the real scenario: the importance of having the doctor close to home sunk for the virtual visit and having a recommendation for doctor in a virtual doctor's visit was reported as less important than for a regular visit.

When focusing on the conditions which should be given that a virtual visit would be full accepted, there are some key claims: data safety, an cautionary handling of data access, and the claim that the costs must be covered by insurance funds. Moreover, a good usability is required, an easy organization, a high learnability, and ease of use of the technical systems.

Especially when acceptance of technical systems in this sensitive field is studied, an explicit focus should be directed to users' diversity. As opposed to the past, when mostly sophisticated and technology prone professionals were typical end-users of technical products, now a broader user group has access to technology in general. This is especially crucial in medical technology as mostly old and technology inexperienced persons will be confronted with the need to use medical technology. Therefore, we looked at different user factors in their impact on the acceptance of telemedical applications. It is remarkable that-besides some singular age effects-age mostly did not impact the reported attitudes. In contrast to outcomes in many other fields of technology usage, in which there are distinct differences between younger and older adults, this is not the case in this context. Also no gender effects on acceptance were revealed. This shows that the different cognitive mindsets of technology of older adults as well as their lower technical expertise are not the crucial player for acceptance outcomes here. In addition, the different styles in dealing with own health did not show a large impact on acceptance. As the most prominent factor, respondents' perception of their competence when dealing with technology did show the most influence. Thus, it should be an explicit aim to support users in frequently dealing with technology in order to positively influence individual self-competence levels.

\section{ACKNOWLEDGMENTS}

We would like to thank our interview partners, who volunteered to take part in this study, as well as the senior home St. Peter and the friendly people living and working there. This work was funded by the Excellence Initiative of German State and Federal Government.

\section{REFERENCES}

[1] H. R. Behrendt (2009). Ein Problemaufriss über den demographischen Wandel in der Bundesrepublik Deutschland. Notfall \& Rettungsmedizin, $12(1), 45-50$

[2] S. M. Prückner (2009). Der demographische Wandel. Notfall \& Rettungsmedizin, 12 (1), 13-18.

[3] R. L. Bashshur, T. G. Reardon, and G. W. Shannon, "Telemedicine: A New Health Care Delivery System", Annual Review of Public Health, vol. 21, 2000, pp.613-637.

[4] P. Whitten, and B. Love, "Patient and provider satisfaction with the use of telemedicine: Overview and rationale for cautious enthusiasm", Journal of Postgrad Medicine, vol. 51, 2005, pp.294-300.

[5] S. Beul, S. Mennicken, M. Ziefle, E.-M. Jakobs, "What Happens After Calling the Ambulance: Information, Communication, and Acceptance
Issues in a Telemedical Workflow". In C.A Shoniregun and G.A. Akmayeva (eds.). Proceedings of the International Conference on Information Society, 2010, London: Infonomics Society, pp. 111-116.

[6] M. Ziefle, S. Beul, S. Mennicken and E.-M. Jakobs, " Communication and Information Barriers in Telemedical Applications in Emergency Situations - Emergency Doctors' Point of View. International Journal for Digital Society, vol. 2(1), in press.

[7] J. Preston, F.W.Brown, and B. Hartley, "Using telemedicine to improve health care in distant areas", Hospital and Community Psychatry, vol. 43/1), 1992, pp.25-32.

[8] R. Holle, and G. Zahlmann, "Evaluation of telemedical services", IEEE Transactions on Information Technology in Biomedicine, vol. 3(2), 1999, pp.84-91

[9] P. J. Hu, P. Y. K. Chau, O. R. Liu Sheng, and K. Yan Tam, “ Examining the technology acceptance model using physician acceptance of telemedicine technology", Journal of Management Information Systems, vol. 16 (2), 1999 , pp.91-112.

[10] J. E. Stahl, and R. F. Dixon, "Acceptability and willingness to pay for primary care videoconferencing: a randomized controlled trial Journal of Telemedicine and Telecare, vol. 16, 2010, pp.147-151.

[11] C. Azzolini (2011): A pilot teleconsultation network for retinal diseases in ophthalmology J Telemed Telecare 17(1): 20-24;

[12] T. Johansson, C. Wild 2011 Telerehabilitation in stroke care- a systematic review, International Journal of Telemedicine and Telecare, Volume 17, Number 1, pp. 1-6

[13] L. Eriksson, B. Lindström, and L. Ekenberg, Patients' experiences of telerehabilitation at home after shoulder joint replacement $\mathrm{J}$ Telemed Telecare 17(1): 25-30;

[14] S. Buck, "Nine Human Factors Contributing to the User Acceptance of Telemedicine Applications: A Cognitive-Emotional Approach", Journal of Telemedicine \& Telecare, vol. 15, no. 2, 2009, pp.55-58.

[15] K. Jungyoon, J. L. DelliFraine, K. H. Dansky, K. J. McCleary 2010 Physicians' acceptance of telemedicine technology: an empirical test of competing theories International Journal of Information Systems and Change Management, Volume 4 Issue 3

[16] K. Arning, and M. Ziefle, "Different Perspectives on Technology Acceptance: The Role of Technology Type and Age", Human Computer Interaction for eInclusion. LNCS 5889, A. Holzinger and K. Miesenberger, Eds. Berlin, Heidelberg: Springer, 2009, pp.20-41.

[17] J. C. Flanagan, "The critical incident technique. Psychological Bulletin“, vol. 51, 1954, pp.327-359.

[18] J.E. Stahl and R.F. Dixon (2010). Acceptability and willingness to pay for primary care videoconferencing: a randomized controlled trial"

[19] S. Gaul and M. Ziefle. "Smart Home Technologies: Insights into Generation-Specific Acceptance Motives". Human - Computer Interaction for eInclusion. LNCS 5889, A. Holzinger and K. Miesenberger, Eds. Berlin, Heidelberg: Springer, 2009, pp.312-332.

[20] F. D. Davis, "Perceived Usefulness, Perceived Ease of Use, and User Acceptance of Information Technology", MIS Quarterly, vol. 13, 1989, pp.319-340.

[21] M. Ziefle and W. Wilkowska, "Technology acceptability for medical assistance," in Proceedings of the $4^{\text {th }}$ ICST/IEEE Conference on Pervasive Computing Technologies for Healthcare, 2010, DOI 10.4108/ICST.PERVASIVEHEALTH2010.8859.

[22] W. Wilkowska and M. Ziefle, "User diversity as a challenge for the integration of medical technology into future home environments," in Human-Centered Design of eHealth Technologies: Concepts, Methods and Applications, M. Ziefle and C. Röcker, Eds. Hershey, PA, USA: IGI Global, 2011, pp. 95-126.

[23] V. Venkatesh, M. G. Morris, G. B. Davis, and F. D. Davis, "User acceptance of information technology: Toward a unified view", MIS Quarterly, vol. 27, no. 3, 2003, pp.425-478. 